\title{
Variety is the Splice of Life
}

\section{Mukesh K. Jain and Anne Hamik}

The circulatory system is the first functioning organ system to develop in vertebrate embryos, and for mammalian embryos its function is critical for early embryo survival (1). Coordinated development of the heart, the blood vessels, and the blood requires close communication among the cells making up the different components (2), with nuclear events constituting the final common pathway of the networks that determine cell function and phenotype. During development in particular, the precise control of spatial and temporal expression of mRNAs and their protein products is required for successful system formation.

Regulation of transcription factor (TF) activity is integral for this precision. Numerous studies have demonstrated lethal consequences of loss, abnormal function, or dysregulation of the temporal and cell-specific activity of TFs during vascular development $(3,4)$. Precise regulation of TF activity can be achieved by alterations at several levels, including chromatin modifications, the concomitant presence of co-activating or inhibitory proteins, and regulation of expression of the TF itself, to name a few. Of the various mechanisms, regulation of TF expression via alternative splicing provides a potent source for precise regulation by generating a large number of protein products, from a single gene, that can have unique temporal- and tissue-specific functions.

Alternative splicing (AS), the process by which the exons of primary transcripts (pre-mRNAs) from genes can be spliced in different arrangements to produce structurally and functionally distinct mRNA and protein variants, is used extensively to create greater macromolecular and cellular complexity of higher eukaryotic organisms (5). In fact, this mechanism is responsible for producing 5-10 times more cellular proteins than expected from the 30,000 genes inferred from the human genome sequence (6). Variant transcripts generated from AS, similar to those initiated from distinct promoters, are often tissue and/or developmental specific, resulting in effects seen only in certain cells or developmental stages (7).

The role of AS in cellular complexity extensive; over $75 \%$ of human genes contain one or more alternative exons (5). It is estimated that there are between one and two AS events per multiintron gene, the majority of which are biologically significant because they affect the coding sequence (8). Indeed, TFs may have an even a higher rate of alternatively-spliced isoforms than do other genes. Taneri et al. created a database of splice variants in the mouse transcriptosome, and found that $29 \%$ of all loci demonstrated variant exons. TF loci $(n=461)$ showed variant exons in $62 \%$ (9). Tissue-specific isoforms of TF were demonstrated, consistent with a major role of AS in mediating TF activity and cell differentiation.

Frequently, AS does not directly affect DNA binding but modulates various other characteristics of TF function. Alternatively-spliced isoforms may result in the introduction of stop codons (leading to degradation of mRNA by nonsense-mediated decay, or can differ in

\footnotetext{
Publisher's Disclaimer: This is a PDF file of an unedited manuscript that has been accepted for publication. As a service to our customers we are providing this early version of the manuscript. The manuscript will undergo copyediting, typesetting, and review of the resulting proof before it is published in its final citable form. Please note that during the production process errors may be discovered which could affect the content, and all legal disclaimers that apply to the journal pertain.
} 
the formation or composition of complexes between various transcription factors, intracellular localization, altered protein stability or TF expression levels, or lead to the addition of new protein parts, including posttranslational modification (7). Finally, TFs acting at the level of initiation and elongation can impact splice-site selection $(5,10)$, adding to the complexity of AS in control of TF function.

Recent studies highlight the importance of AS in cardiovascular biology. For example, serum response factor (SRF) is a widely expressed TF that regulates muscle differentiation and cellular proliferation, including growth programs in cardiac and vascular smooth muscle cells. SRF is known to differentially regulate the gene sets active in differentiation or proliferation via several mechanisms including regulated expression and DNA binding, association with cell-restricted cofactors, and alternative splicing (11). Recently, novel SRF isoforms were identified which demonstrate differential functions. Isoforms that lack the activating domain still bind to DNA, but act as repressors of SRF target genes, including SRF itself $(12,13)$. Another isoform contains a premature termination codon, leading to nonsense-mediated mRNA decay of the transcript. Interestingly, the number of different SRF isoforms appears to increase in humans as they age, with isoforms deficient in activating function increasing in relative proportion (13). Thus, AS results in three independent mechanisms for regulating SRF function-negative feedback, transcript decay, and changes in relative abundance of isoforms.

Myocardin is a cardiac- and smooth muscle-specific TF that functions as a co-activator for SRF. Alternative splicing of myocardin produces myocardin transcripts that initiate translation at two different ATG codons located 79 amino acids apart (14). Both isoforms contain a basic $(+)$ region that mediates binding to and activation of SRF. The longer myocardin isoform, predominant in cardiac muscle, also has an extreme $\mathrm{N}$-terminal domain that confers the ability to bind to MEF2, a TF that also controls growth-associated genes in heart and vascular smooth muscle. Conservation of the 5' and 3' splice sites across vertebrate genomes suggests that this splicing event is an evolutionarily conserved mechanism allowing myocardin to stimulate MEF2 activity in the heart (14).

The coordinated, sequential activation of various genes required to successfully direct angiogenesis is controlled by TFs such as RUNX2. RUNX2 is required for normal vascular development in mice (15) and acts via induction of expression of vascular endothelial cell growth factor (VEGF) (16), critical in the initial activation stage of angiogenesis. Later stages of vessel formation involve vessel remodeling and apoptosis, largely regulated by transforming growth factor $\beta$ (TGF $\beta$ ). In proliferating endothelial cells and aortic rings undergoing vascular sprouting, RUNX2 and a RUNX2 isoform, RUNX2 8 , are expressed (17). RUNX2, but not RUNX2 $\delta 8$, confers resistant to growth inhibition by TGF $\beta$, suggesting the alternative splicing of RUNX2 regulates the angiogenic process by affecting the function of TGF $\beta$ (17). These results illustrate the widespread phenomenon of developmentally regulated isoforms of TF, which, in turn, regulate development.

In this issue of JMCC, Wu et al. describe novel characteristics of a recently identified TF, PRDM6, and present data suggesting it has a role in vascular precursor differentiation. By identifying multiple full length isoforms of PRDM6 in hematovascular precursor cells and endothelial cell lines, this study builds on the initial description of PRDM6 as a smooth musclerestricted epigenetic regulator (18) and establishes isoform- and cell- specific functions of PRDM6.

The PR domain (PRDM) proteins are a small subfamily of the Kruppel-like zinc finger DNA binding transcription factors. The Kruppel-like family of zinc finger genes is characterized by Cys2-His2 zinc fingers and can be divided further into subsets based on other structural features, including a seven-amino acid $\mathrm{H} / \mathrm{C}$ link between adjacent fingers and several 
conserved NH2-terminal modules (19). The PRDM family was first identified when a homologous N-terminal 100-amino acid region (PR domain) was recognized as shared by RIZ (retinoblastoma protein-interacting zinc finger; PRDM2) and the PRDI-BF1/BLIMP1 transcription repressor (PRDM1) that promotes B lymphocyte maturation (20). To date, $\sim 20$ PR domain family members have been identified. All studies thus far show these factors to have growth suppressive activities, and several are silenced in a variety of human cancers $(21,22)$.

Characteristic of the PR domain family of transcription factors is the presence of multiple isoforms. For example, proteins are translated from two PRDM1 isoform variants, RIZ1 and RIZ2. RIZ1 has tumor suppressor activity (23). Levels of RIZ1 are substantially decreased in breast, neuroblastoma, colorectal, gastric, endometrial and lung cancers, and it is targeted for inactivation by various genetic and epigenetic mechanisms, including deletion, frameshift mutations, promoter hypermethylation, missense mutations, and splicing aberrations (21). RIZ2, however, is variously described as normally expressed in primary tumors (23) or overexpressed in tumor cells (24), and appears to be oncogenic, conferring an increased growth rate, insensitivity to inhibitory signals, and a higher level of expression of cyclin D1 and A (25). The two isoforms differ solely by the presence (RIZ1) or absence (RIZ2) of the N-terminal PR domain (26). The PR domain mediates protein-protein interactions (27) and is associated with histone methyltransferase activity (24), while the zinc finger domain mediates the putative oncogenic activity of RIZ2 (25).

The study by Wu et al. also demonstrates an isoform-specific effect of PRDM proteins on cell proliferation. PRISM/PRDM6, as described by Davis et al. (18), suppresses differentiation and maintains the proliferative potential of smooth muscle cells, whereas the PRDM6 isoform assessed by Wu et al. (PRDM6/4\#) inhibits endothelial cell proliferation, and does not affect smooth muscle cell proliferation. The two isoforms differ only by the presence of an additional 170 residues at the amino terminal of PRDM6/4\#. The differential effects of PRDM6 on proliferation in SMC and EC are most likely associated with the cell-specific expression of PRDM6 isoforms. Thus, just within the PRDM subfamily of zinc-finger TF, there are examples illustrating that the regulation of TF isoforms can lead to modulation of the number of copies of DNA binding motifs, DNA binding affinity or specificity, generation of transcriptional enhancers and their antagonists from the same gene, and opposing effects in different cell types.

AS of TF transcripts has marked effects on complex processes such as cardiac and vascular development. Classically, studies of TF activation have consisted largely of assessment of expression levels of TF and of the effects of TF over-expression. However, the majority of genes regulated in a tissue-specific manner by AS are different from those regulated in a tissuespecific manner at the transcriptional level (5). As discussed above, AS of TF appears to be a common event. Moreover, there is emerging evidence that groups of tissue-specific AS events may function in a coordinated manner in specific pathways or interaction networks, in much the same way as has been observed for groups of genes co-regulated at the transcriptional level (5). As many as 50\% of disease mutations in exons may impact on splicing, attesting to the importance in introducing routine transcript analyses in order to properly assess possible mechanisms accounting for human diseases (5). The use of AS microarray profiling technologies will allow acquisition of AS signatures and detection of disease-relevant changes in gene expression at the level of splicing (5). Thus, more sophisticated assessment of TF activity- targeting individual alternative exons when attempting to understand the regulation of complex processes- will be need to become a common practice. 


\section{REFERENCES}

1. Copp AJ. Death before birth: clues from gene knockouts and mutations. Trends Genet 1995;11(3):8793. [PubMed: 7732578]

2. Ema M, Rossant J. Cell fate decisions in early blood vessel formation. Trends Cardiovasc Med 2003;13 (6):254-9. [PubMed: 12922023]

3. Hamik A, Wang B, Jain MK. Transcriptional regulators of angiogenesis. Arterioscler Thromb Vasc Biol 2006;26(9):1936-47. [PubMed: 16778118]

4. Olson EN. Gene regulatory networks in the evolution and development of the heart. Science 2006;313 (5795):1922-7. [PubMed: 17008524]

5. Blencowe BJ. Alternative splicing: new insights from global analyses. Cell 2006;126(1):37-47. [PubMed: 16839875]

6. Finishing the euchromatic sequence of the human genome. Nature 2004;431(7011):931-45. [PubMed: 15496913]

7. Stamm S, Ben-Ari S, Rafalska I, Tang Y, Zhang Z, Toiber D, et al. Function of alternative splicing. Gene 2005;344:1-20. [PubMed: 15656968]

8. Gupta S, Zink D, Korn B, Vingron M, Haas SA. Genome wide identification and classification of alternative splicing based on EST data. Bioinformatics 2004;20(16):2579-85. [PubMed: 15117759]

9. Taneri B, Snyder B, Novoradovsky A, Gaasterland T. Alternative splicing of mouse transcription factors affects their DNA-binding domain architecture and is tissue specific. Genome Biol 2004;5 (10):R75. [PubMed: 15461794]

10. Kornblihtt AR. Chromatin, transcript elongation and alternative splicing. Nat Struct Mol Biol 2006;13 (1):5-7. [PubMed: 16395314]

11. Miano JM. Serum response factor: toggling between disparate programs of gene expression. J Mol Cell Cardiol 2003;35(6):577-93. [PubMed: 12788374]

12. Belaguli NS, Zhou W, Trinh TH, Majesky MW, Schwartz RJ. Dominant negative murine serum response factor: alternative splicing within the activation domain inhibits transactivation of serum response factor binding targets. Mol Cell Biol 1999;19(7):4582-91. [PubMed: 10373507]

13. Zhang X, Azhar G, Huang C, Cui C, Zhong Y, Huck S, et al. Alternative splicing and nonsensemediated mRNA decay regulate gene expression of serum response factor. Gene. 2007

14. Creemers EE, Sutherland LB, Oh J, Barbosa AC, Olson EN. Coactivation of MEF2 by the SAP domain proteins myocardin and MASTR. Mol Cell 2006;23(1):83-96. [PubMed: 16818234]

15. Li QL, Ito K, Sakakura C, Fukamachi H, Inoue K, Chi XZ, et al. Causal relationship between the loss of RUNX3 expression and gastric cancer. Cell 2002;109(1):113-24. [PubMed: 11955451]

16. Zelzer E, Glotzer DJ, Hartmann C, Thomas D, Fukai N, Soker S, et al. Tissue specific regulation of VEGF expression during bone development requires Cbfa1/Runx2. Mech Dev 2001;106(1-2):97106. [PubMed: 11472838]

17. Sun L, Vitolo MI, Qiao M, Anglin IE, Passaniti A. Regulation of TGFbeta1-mediated growth inhibition and apoptosis by RUNX2 isoforms in endothelial cells. Oncogene 2004;23(27):4722-34. [PubMed: 15107836]

18. Davis CA, Haberland M, Arnold MA, Sutherland LB, McDonald OG, Richardson JA, et al. PRISM/ PRDM6, a transcriptional repressor that promotes the proliferative gene program in smooth muscle cells. Mol Cell Biol 2006;26(7):2626-36. [PubMed: 16537907]

19. Atkins GB, Jain MK. Role of Kruppel-like transcription factors in endothelial biology. Circ Res 2007;100(12):1686-95. [PubMed: 17585076]

20. Buyse IM, Shao G, Huang S. The retinoblastoma protein binds to RIZ, a zinc-finger protein that shares an epitope with the adenovirus E1A protein. Proc Natl Acad Sci U S A 1995;92(10):446771. [PubMed: 7538672]

21. Canote R, Du Y, Carling T, Tian F, Peng Z, Huang S. The tumor suppressor gene RIZ in cancer gene therapy (review). Oncol Rep 2002;9(1):57-60. [PubMed: 11748455]

22. Jiang GL, Huang S. The yin-yang of PR-domain family genes in tumorigenesis. Histol Histopathol 2000;15(1):109-17. [PubMed: 10668202] 
23. He L, Yu JX, Liu L, Buyse IM, Wang MS, Yang QC, et al. RIZ1, but not the alternative RIZ2 product of the same gene, is underexpressed in breast cancer, and forced RIZ1 expression causes G2-M cell cycle arrest and/or apoptosis. Cancer Res 1998;58(19):4238-44. [PubMed: 9766644]

24. Derunes C, Briknarova K, Geng L, Li S, Gessner CR, Hewitt K, et al. Characterization of the PR domain of RIZ1 histone methyltransferase. Biochem Biophys Res Commun 2005;333(3):925-34. [PubMed: 15964548]

25. Rossi M, Abbondanza C, D'Arcangelo A, Gazzerro P, Medici N, Moncharmont B, et al. The Znfinger domain of RIZ protein promotes MCF-7 cell proliferation. Cancer Lett 2004;215(2):229-37. [PubMed: 15488642]

26. Liu L, Shao G, Steele-Perkins G, Huang S. The retinoblastoma interacting zinc finger gene RIZ produces a PR domain-lacking product through an internal promoter. J Biol Chem 1997;272(5):298491. [PubMed: 9006946]

27. Huang S, Shao G, Liu L. The PR domain of the Rb-binding zinc finger protein RIZ1 is a protein binding interface and is related to the SET domain functioning in chromatin-mediated gene expression. J Biol Chem 1998;273(26):15933-9. [PubMed: 9632640] 